\title{
Association of FTO rs9939609 with Obesity in the Kuwaiti Population: A Public Health Concern?
}

\author{
Ahmad Al-Serria Suzanne A. Al-Bustan ${ }^{b}$ Maisa Kamkarc Daisy Thomas ${ }^{c}$ \\ Osama Al-Smaidic Rabeah Al-Temaimia ${ }^{\mathrm{c}}$ Olusegun A. Mojiminiyi ${ }^{d}$
}

Nabila A. Abdella ${ }^{e}$

${ }^{a}$ Human Genetics Unit, Department of Pathology, Faculty of Medicine, Kuwait University, Safat, Kuwait;

${ }^{\mathrm{b}}$ Department of Biological Sciences, Faculty of Science, Kuwait University, Safat, Kuwait; ' $G e n e t i c s$ and Genomics Unit, Research Division, Dasman Diabetes Institute, Safat, Kuwait; ${ }^{d}$ Department of Pathology, Faculty of Medicine,

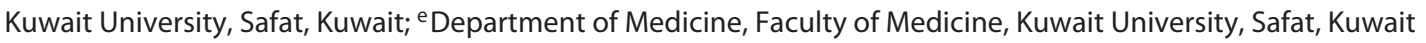

\section{Significance of the Study}

- The common FTO gene polymorphism (rs9939609) is found to be associated with overweight/obesity in an obesogenic population. This highlights the role of genetic variants in increasing the risk of obesity in such a population, hence encouraging researchers to further investigate and elucidate the mechanism involved in the development of obesity.

\section{Keywords}

Obesity · Kuwait · FTO · Polymorphism - Body mass index · Obesogenic population

\begin{abstract}
Objective: To investigate the effect of the common fat mass and obesity-associated (FTO) gene polymorphism rs9939609 on body mass index (BMI) in one of the most obese populations worldwide. Subjects and Methods: Genotypic data for FTO rs9939609 were available for 1,034 unrelated Kuwaiti adults obtained from Kuwait's Dasman Diabetes Institute and Kuwait University. The association between the FTO polymorphism with BMI as continuous and categorical (normal BMI [<25] vs. overweight/obese [ 25]) variables was analyzed using both linear and logistic regression models, re-
\end{abstract}

spectively, with the assumption of both dominant and additive genetic models performed using the SNPassoc package from $R$ statistics. Results: The $A$ allele was associated with increased $\mathrm{BMI}(\beta=1.21 ; 95 \% \mathrm{Cl}=0.16-2.26 ; p=0.023)$. In concordance, the categorical BMI (normal vs. overweight/ obese) also showed a significant association between the $A$ allele and overweight/obesity $(\mathrm{OR}=1.47 ; 95 \% \mathrm{Cl}=1.01-2.12$; $p=0.041$ ). However, no association between the FTO variant was observed with cardiometabolic traits. Conclusion: We observed an association between the common FTO rs9939609 polymorphism and increased BMI (overweight/ obesity) in Kuwaiti adults, which is consistent with previous research in other populations. Our findings encourage further investigation of genetic variants to elucidate the mechanisms involved in the development of obesity in such an obesogenic population.

(C) 2018 The Author(s)

Published by S. Karger AG, Basel

\begin{tabular}{ll}
\hline KARGER & $\begin{array}{l}\text { ○ } 2018 \text { The Author(s) } \\
\text { Published by S. Karger AG, Basel }\end{array} \quad \begin{array}{l}\text { Karger } \\
\text { Open access }\end{array}$ \\
$\begin{array}{l}\text { E-Mail karger@karger.com } \\
\text { www.karger.com/mpp }\end{array}$ & $\begin{array}{l}\text { This is an Open Access article licensed under the Creative Commons } \\
\text { Attribution-NonCommercial-4.0 International License (CC BY-NC) } \\
\text { (http://www.karger.com/Services/OpenAccessLicense), applicable to } \\
\text { the online version of the article only. Usage and distribution for } \\
\text { commercial purposes requires written permission. }\end{array}$
\end{tabular}

Ahmad Al-Serri

Human Genetics Unit, Department of Pathology

Faculty of Medicine, Kuwait University

PO Box 24923, Safat 13110 (Kuwait)

E-Mail Ahmad.Alserri@hsc.edu.kw 


\section{Introduction}

Kuwait has been reported as one of the countries with the highest prevalence of obesity with approximately $50 \%$ of the population reported as being obese [1]. Obesity and decreased physical activity are factors contributing to the prevalence of type 2 diabetes (T2D). The International Diabetes Federation has recently ranked Kuwait among the top 10 countries with a high T2D prevalence of approximately $20 \%$, which could be attributed to the high prevalence of obesity [2].

Genetic factors increase the susceptibility to obesity [3]. In 2007, Frayling et al. [4] reported the fat mass and obesity-associated (FTO) gene as the first genome-wide association study obesity susceptibility gene. Initially, the genome-wide association study showed that the FTO variant (rs9939609) was associated with T2D. However, no association was observed when controlling for body mass index (BMI) [4]. It had been reported that the common variant rs9939609, found in the first intron of the FTO gene, is one of the most commonly replicated polymorphisms associated with obesity in both children and adults [4]. Although the association has been reported in all age groups and in both genders, inconsistency exists with studies supporting the association as being age- and gender-dependent $[5,6]$. The association between FTO variants and BMI has also been reported to interact with both physical activity [7] and dietary intake [8], with physical activity attenuating the impact of FTO on BMI. FTO variants have also been reported to be associated with obesity in different ethnic backgrounds $[5,6]$. Hence, the association between FTO and obesity could be influenced by multiple factors.

FTO is a nuclear protein that belongs to the a-ketoglutarate-dependent, nonheme iron oxygenase superfamily [9]. The gene encodes a 2-oxoglutarate and $\mathrm{Fe}(\mathrm{II})$-dependent demethylase, an enzyme involved in demethylation of deoxyribonucleic acid (DNA) and ribonucleic acid (RNA) $[10,11]$. Studies have shown that the FTO gene is ubiquitously expressed, thereby indicating its involvement in multiple organ systems $[11,12]$. These observations were further studied using mouse models that indicated a role for FTO in the nervous and cardiovascular systems [12]. Elucidating the genetics of obesity may allow for future predictability of this disease which may help in identifying those at high genetic risk of obesity and preventing it through dietary interventions [13].

Due to the high prevalence of obesity in Kuwait and the obesogenic environment in which the population lives, the objective of this study was to assess the prevalence and effect of the common FTO gene polymorphism (rs9939609) on BMI in a cohort representing a Kuwaiti population.

\section{Subjects and Methods}

\section{Study Subjects and Ethics Statement}

A total of 1,034 subjects with FTO rs9939609 genotypic data were obtained from 2 institutions: Kuwait University and Dasman Diabetes Institute, Kuwait. Exclusion criteria were children aged $<18$ years and non-Kuwaitis. Hence, a total of 888 subjects were available for analysis. All the subjects were requested to participate in the study during outpatient visits to Mubarak Al Kabeer Hospital, Kuwait, and the Dasman Diabetes Institute's Genetic Clinic. The Ethics Committees at the Ministry of Health, the Faculty of Medicine, and the Office of Research Affairs at Dasman Diabetes Institute approved the study which adheres to the Declaration of Helsinki on ethical principles for medical research involving human subjects. The study was explained to all participants, and both written and oral informed consents were obtained for this study. Similarly, consents from parents were provided for subjects $<18$ years of age. Only subjects who gave consent to participate in the study were included and requested to provide two 5-mL fasting EDTA blood samples. All T2D patients $(n=294)$ were under treatment with oral hypoglycemic agents, and none were on insulin or insulin-sensitizing drugs (thiazolidinedione compounds) or lipidlowering medication at the time of recruitment.

\section{Anthropometric Measurements}

Every participant in this study underwent a detailed history and medical examination for anthropometric assessment (Table 1). All anthropometric measurements were made by trained observers and/or clinical research coordinators using standard techniques with the participants wearing light clothes without shoes. Height was measured to the nearest $0.1 \mathrm{~cm}$ using a stadiometer and weight to the nearest $0.1 \mathrm{~kg}$ using a standardized standing beam balance. Then, BMI was calculated as weight (kilograms) per squared height (square meters) and categorized based on WHO standards (underweight: $>18.5$, normal: $<25$, overweight: $\geq 25$, and obese: $\geq 30$ ) [14]. In addition, the 2 fasting EDTA blood samples collected from each participant were used for biochemical analysis using plasma, and for genomic DNA extraction. Standard routine fasting plasma glucose, total cholesterol, triglycerides, and high-density lipoprotein cholesterol were analyzed on an automated analyzer (Beckman Unicel DxC 800, Beckman Corporation, Brea, CA, USA). Low-density lipoprotein cholesterol was calculated using the Friedewald formula [15].

\section{DNA Isolation and Genotyping}

DNA was extracted from $200 \mu$ l of whole blood using a Qiagen DNA mini kit (Qiagen, CA, USA) according to the manufacturer's instructions [16]. The TaqMan genotyping assay (Life Technologies, CA, USA) was used to determine the FTO variant rs9939609 genotypes according to standard manufacturer protocols [17]. Allelic discrimination was performed and analyzed using ABI 7500 fast real-time PCR system SDS software (Life Technologies, CA, USA). 
Table 1. Anthropometric and metabolic characteristics, and FTO rs9939609 genotype frequencies, according to body mass index category

\begin{tabular}{lcccc}
\hline Variable & $\begin{array}{l}\text { Normal weight } \\
(n=214)\end{array}$ & $\begin{array}{l}\text { Overweight/obese } \\
(n=674)\end{array}$ & $\begin{array}{l}\text { All participants } \\
(n=888)\end{array}$ & $p$ \\
\hline Age, years & $29.3 \pm 15.5$ & $45.5 \pm 16.3$ & $41.6 \pm 17.6$ & $<0.0001$ \\
Subjects (female) & $142(66 \%)$ & $417(62 \%)$ & $559(63 \%)$ & 0.256 \\
BMI & $21.9 \pm 2$ & $33.3 \pm 6.8$ & $30.5 \pm 7.7$ & $<0.0001$ \\
Total cholesterol, mmol/L & $4.5 \pm 1.3$ & $4.8 \pm 1.06$ & $4.7 \pm 1.14$ & 0.009 \\
LDL-C, mmol/L & $2.8 \pm 1.34$ & $2.9 \pm 0.95$ & $2.9 \pm 1.04$ & 0.752 \\
Triglyceride, mmol/L & $0.7 \pm 0.48$ & $1.5 \pm 1.44$ & $1.3 \pm 1.33$ & $<0.0001$ \\
HDL-C, mmol/L & $1.3 \pm 0.34$ & $1.1 \pm 0.35$ & $1.1 \pm 0.35$ & 0.0003 \\
FPG, mmol/L & $7.2 \pm 3.9$ & $7.6 \pm 3.6$ & $7.6 \pm 3.6$ & 0.419 \\
T2D (yes) & $24(11 \%)$ & $270(40 \%)$ & $294(33 \%)$ & $<0.0001$ \\
FTO subtypes & & & & 0.147 \\
TT & $71(33 \%)$ & $187(27.5 \%)$ & $258(29 \%)$ & $426(48 \%)$ \\
$\quad$ TA & $101(47 \%)$ & $325(48.5 \%)$ & $204(23 \%)$ & \\
AA & $42(20 \%)$ & $162(24 \%)$ & & \\
\hline
\end{tabular}

BMI, body mass index; HDL-C, high-density lipoprotein cholesterol; LDL-C, low-density lipoprotein cholesterol; FPG, fasting plasma glucose; T2D, type 2 diabetes. If percentages are not indicated, the values given indicate means \pm standard deviations.

\section{Statistical Analysis}

Statistical analysis was performed using the Statistical Package for the Social Sciences software (version 23; SPSS Inc., Chicago, IL, USA). Results were expressed as mean \pm standard deviation, and percentages where appropriate. Hardy-Weinberg equilibrium was tested using the web-based calculator available at http://www.oege. org/software/hwe-mr-calc.shtml (accessed on December 18, 2017) [18]. Analysis of variance was used to assess the association between the FTO variant and continuous variables. The Pearson $\chi^{2}$ test was used for the analysis of categorical variables. The association of the FTO variant and categorical BMI was assessed by binary logistic regression represented as odds ratio (OR) and 95\% confidence intervals (CI) after adjusting for both age and gender. Linear regression was used to assess the association between the FTO variant and BMI as a continuous variable represented by the $\beta$-coefficient and 95\% CI after adjusting for both age and gender. We considered 2 genetic models: a dominant model (TT vs. TA and AA) to assess the impact of carrying at least $1 \mathrm{~A}$ allele compared to none and an additive model to assess the dose-dependent association of the A allele using the SNPassoc package from $R$ software [19]. Significance was set as $p<0.05$.

\section{Results}

\section{Population Characteristics}

Our population sample included 888 Kuwaiti nationals, of whom 559 were females and 329 were males. The characteristics of the population studied are given in Table 1 . Analysis was based on subjects being split into 2
Table 2. Body mass index category distribution according to gender

\begin{tabular}{lcccc}
\hline & $\begin{array}{l}\text { All partici- } \\
\text { pants, } n(\%)\end{array}$ & $\begin{array}{l}\text { Males, } \\
n(\%)\end{array}$ & $\begin{array}{l}\text { Females, } \\
n(\%)\end{array}$ & $p$ \\
\hline Underweight & $14(1.6)$ & $2(0.6)$ & $12(2.1)$ & 0.006 \\
Normal & $200(22.5)$ & $70(21.3)$ & $130(23.3)$ & \\
Overweight & $260(29.3)$ & $117(35.6)$ & $143(25.6)$ & \\
Obese & $414(46.6)$ & $140(42.5)$ & $274(49.1)$ & \\
\hline
\end{tabular}

binary categories representing normal weight subjects with BMI $<25$ and overweight/obese subjects with BMI $\geq 25$. Overweight and obese subjects represent $n=674$ (76\%) as opposed to $n=216$ (24\%) being normal. Increase in age was significantly associated with overweight/obesity. Cardiometabolic traits were significantly different between normal and overweight/obese subjects $(p<0.01)$. The FTO rs9939609 genotype frequencies were in HardyWeinberg equilibrium $(p>0.05)$ and are presented in Table 1 . The frequency of the A allele was found to be $47 \%$. T2D is significantly more common in overweight and obese subjects $(40 \%)$ compared to normal BMI subjects (11\%), with $p<0.0001$. We did not observe any difference between males and females when categorically comparing normal versus overweight and obese BMI $(p=0.256)$ 
Table 3. Relation between FTO genotypes and anthropometric and metabolic characteristics

\begin{tabular}{|c|c|c|c|c|}
\hline Variable & $\mathrm{TT}(n=258)$ & TA $(n=426)$ & $\mathrm{AA}(n=204)$ & $p$ \\
\hline Age, years & $42.6 \pm 18.3$ & $40.7 \pm 16.8$ & $42.4 \pm 18.1$ & 0.307 \\
\hline Subjects (female) & $153(27 \%)$ & $273(49 \%)$ & $133(24 \%)$ & 0.342 \\
\hline \multicolumn{5}{|l|}{ BMI category } \\
\hline$\leq 24.9^{\circ}$ & $71(27.5 \%)$ & $101(24 \%)$ & $42(20 \%)$ & 0.501 \\
\hline $25-29.9$ & $71(27.5 \%)$ & $124(29 \%)$ & $65(31 \%)$ & \\
\hline$\geq 30$ & $116(45 \%)$ & $201(47 \%)$ & 97 (49\%) & \\
\hline Mean $\mathrm{BMI} \pm \mathrm{SD}$ & $29.8 \pm 7.1$ & $30.8 \pm 8.1$ & $31.1 \pm 7.7$ & 0.08 \\
\hline Total cholesterol, mmol/L & $4.6 \pm 1.1$ & $4.8 \pm 1.2$ & $4.7 \pm 1.1$ & 0.113 \\
\hline $\mathrm{LDL}-\mathrm{C}, \mathrm{mmol} / \mathrm{L}$ & $2.81 \pm 0.9$ & $2.97 \pm 1.1$ & $2.88 \pm 0.8$ & 0.186 \\
\hline Triglyceride, mmol/L & $1.47 \pm 1.2$ & $1.4 \pm 1.5$ & $1.24 \pm 0.6$ & 0.197 \\
\hline $\mathrm{HDL}-\mathrm{C}, \mathrm{mmol} / \mathrm{L}$ & $1.15 \pm 0.3$ & $1.19 \pm 0.3$ & $1.19 \pm 0.3$ & 0.432 \\
\hline $\mathrm{FPG}, \mathrm{mmol} / \mathrm{L}$ & $7.8 \pm 3.7$ & $7.6 \pm 3.8$ & $7.5 \pm 3.2$ & 0.807 \\
\hline T2D (yes) & $92(31 \%)$ & $137(47 \%)$ & $65(22 \%)$ & 0.508 \\
\hline
\end{tabular}

BMI, body mass index; HDL-C, high-density lipoprotein cholesterol; LDL-C, low-density lipoprotein cholesterol; FPG, fasting plasma glucose; T2D, type 2 diabetes. If percentages are not indicated, the values given indicate means \pm standard deviations.

(Table 1); however, differences in the distribution of all BMI categories between males and females were observed ( $p=0.006$; Table 2). There was no difference in mean age between males ( $42.2 \pm 17.7$ years) and females (41.2 \pm 17.5 years): $p>0.05$. Obesity was more prevalent in females compared to males, with $n=274(49.1 \%)$ and $n=140$ (42.5\%), respectively, whereas prevalence of overweight in males was higher compared to that in females, with $n=117(35.6 \%)$ and $n=143(25.6 \%)$, respectively, $p=$ 0.007 . Similarly, when the BMI was used as a continuous variable, a significant difference was observed with females showing a mean BMI of $30.9 \pm 8.4$ compared to a mean BMI of $29.9 \pm 6.4$ in males, with $p=0.04$.

\section{Relationship between FTO rs9939609 and BMI, \\ Cardiometabolic Traits, and T2D Risk}

A $\chi^{2}$ test showed no statistical significance in the distribution of the FTO variant between both normal and overweight/obese BMI categories ( $p=0.147$; Table 3$)$. However, the distribution of the AA genotype was found to be higher $(24 \%)$ in the overweight/obese group compared to the normal BMI group (19.6\%). The TT genotype distribution was found to be higher in the normal BMI group (33.2\%) compared to the overweight/obese group (27.2\%). Using BMI as a continuous variable, we observed a dose-dependent trend with the A allele showing an increased BMI; however, results were not significant $(p=0.08)$ (Fig. 1). The relationships between the FTO variant and cardiometabolic traits and T2D risk

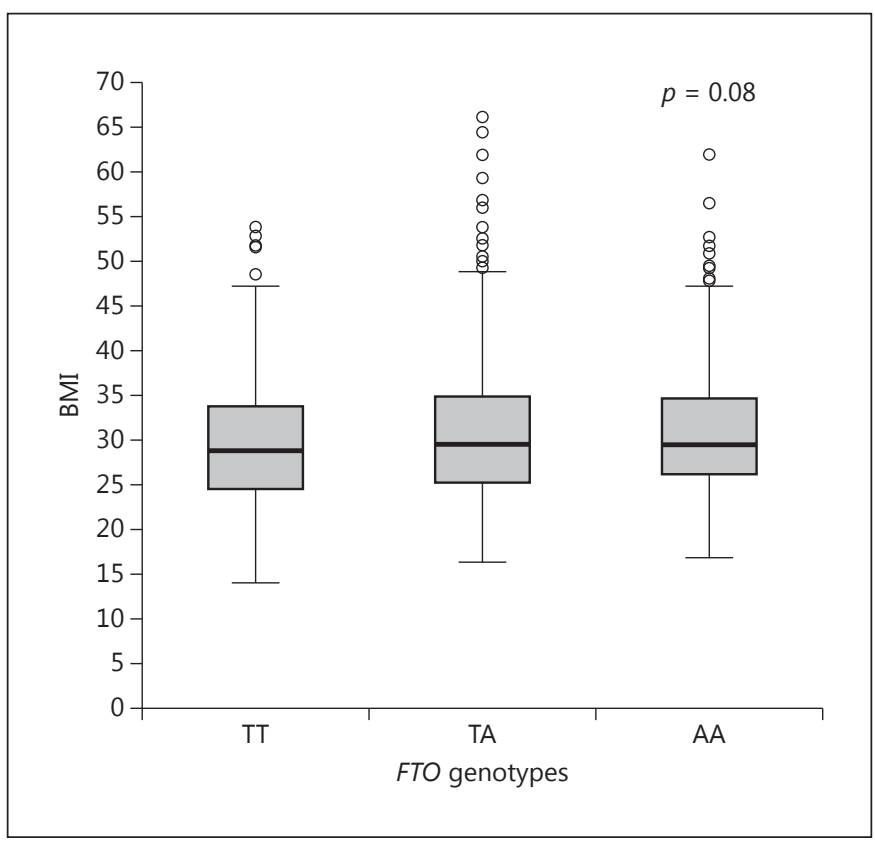

Fig. 1. Boxplot demonstrating the distribution of mean BMI (weight/height ${ }^{2}$ ) for the 3 FTO genotypes (TT, TA, and AA) with an unadjusted $p$ value.

were also evaluated (Table 3). Our results showed no association between the FTO variant and any of the cardiometabolic traits along with T2D prevalence even after controlling for age and gender $(p>0.05)$.
Al-Serri et al. 
Table 4. Logistic regression to predict factors associated with categorical BMI (overweight and obese vs. normal BMI)

\begin{tabular}{lcc}
\hline Variable & OR $(95 \% \mathrm{CI})$ & $p$ \\
\hline Age (+1 year) & $1.06(1.05-1.08)$ & $<0.0001$ \\
Sex (female) & $0.81(0.57-1.16)$ & 0.265 \\
FTO (dominant model) & 1 & 0.041 \\
$\quad$ TT & $1.47(1.01-2.12)$ & \\
$\quad$ TA + AA & \\
\hline
\end{tabular}

Table 5. Linear regression model to predict factors associated with BMI

\begin{tabular}{lcc}
\hline Variable & $\beta(95 \% \mathrm{CI})$ & $p$ \\
\hline Age (+1 year) & $0.15(0.08-0.15)$ & $<0.0001$ \\
Sex (female) & $1.18(0.19-2.16)$ & 0.019 \\
FTO (dominant model) & 1 & 0.023 \\
$\quad$ TT & $1.21(0.16-2.26)$ & \\
TA + AA & & \\
\hline
\end{tabular}

Logistic regression analysis adjusted for age and gender under a genetic dominant model showed carriers of the $\mathrm{A}$ allele were associated with the overweight/obese group $(\mathrm{OR}=1.47 ; 95 \% \mathrm{CI}=1.01-2.12 ; p=0.041)$ (Table 4). Similarly, an additive genetic model showed a significant trend of the A allele with overweight/obesity (OR = $1.29 ; 95 \% \mathrm{CI}=1.02-1.64 ; p=0.036)$. Nevertheless, linear regression analysis using $\mathrm{BMI}$ as a continuous variable showed the A allele to be associated with increased BMI $(\beta=1.21 ; 95 \% \mathrm{CI}=0.16-2.26 ; p=0.023)$ under a dominant genetic model after adjusting for age and gender (Table 5). Although a trend was observed when an additive genetic model was used, results did not reach significance $(\beta=0.62 ; 95 \% \mathrm{CI}=-0.031$ to $1.29 ; p=0.062)$. Age was found to be a significant predictor $(p<0.05)$ in both logistic and linear regressions; however, only gender (female) was found to be a predicting factor of a higher BMI in the linear regression analysis (Table 5).

\section{Discussion}

Our results indicate an association between the common FTO rs9939609 gene polymorphism with overweight/obesity. Prevalence of the high-risk genotype (AA) was found to be higher in the overweight/obese group compared to the normal BMI group. Moreover, the
A allele was associated with increased BMI when used as a continuous variable. We did not observe an association between the FTO rs9939609 with T2D risk. Similarly, we did not find an association with cardiometabolic traits.

Our findings are in concordance with previous studies reporting the association of the FTO A allele with increased BMI and that the frequency of the A allele in our study was similar to that observed in other populations ranging from 45 to $48 \%[4,20]$. This may suggest that the effects of environmental factors on obesity in Kuwait are larger than genetic factors. In addition, this may also suggest that other variants within FTO or other genes may explain the high prevalence of obesity seen in our studied population. It is reported that multiple neighboring single-nucleotide polymorphisms within the first intronic region of the FTO gene are associated with BMI [21]. This is consistent with recent findings showing that different variants within the FTO gene are associated with different classes of obesity [6]. Moreover, a study by Sentinelli et al. [20] showed an association between the common FTO rs9939609 polymorphism with BMI in an Italian population; however, a stronger association was observed with another polymorphism, rs9930506, within the FTO gene suggesting the impact of other polymorphisms on BMI. This also supports findings that the impact of FTO on obesity is population-dependent $[20,22]$. These findings may explain our borderline association of the rs9939609 with the BMI; however, this needs to be replicated further in other cohorts in the population. The FTO variant has been reported to be involved in weight regain after bariatric surgery [23] which is considered a common obesity intervention in the Kuwaiti population [24], and therefore identifying patients at high risk of weight regain prior to intervention may help in planning the course of treatment.

On the other hand, we did not find any association of FTO rs9939609 with the T2D risk. A recent study by Kamura et al. [25] suggested that the association between FTO variants and T2D is mediated through the lifetime maximum BMI at the time of or before diagnosis of T2D. Nevertheless, our lack of association with the T2D risk has also been reported in populations of similar genetic background to the Kuwaiti population [26, 27]. It is apparent that ethnicity plays a role in FTO rs9939609 and T2D risk among different populations, possibly under the influence of other T2D susceptibility factors of stronger effect.

The relation between the FTO variants and FTO gene expression has been investigated; however, results indicate a lack of correlation [28]. The study by Smemo et al. 
[28] demonstrated the connection between FTO variants and the regulation of the homeobox gene IRX3. Irs3-defecient mice demonstrated a $20-25 \%$ reduction in body weight. The study suggests that FTO acts as a transcriptional factor involved in regulating the expression of IRX3 through its interaction with the promoter region of the IRX3 gene which is found to be nearly a megabase away. The relation of the 2 genes is also supported by the high linkage disequilibrium between variants in the FTO and IRX genes [29], suggesting that the FTO gene is a regulator of the IRX3 gene [30]. Moreover, the FTO encodes a 2-oxoglutarate and Fe(II)-dependent demethylase, an enzyme involved in the demethylation of DNA and RNA [9-11]. Physical activity and dietary intake have been shown to alter epigenetic mechanisms [31]. A limitation of our study is the lack of data regarding physical activity which may have influenced the impact of FTO on BMI. A recent study by Awad et al. [32] reported low levels of physical activity in both males and females of the Kuwaiti population with about $40 \%$ reported as being physically inactive. The study by Celis-Morales et al. [7] demonstrated that physical activity attenuates the effect of FTO on BMI, which may suggest an impact of physical inactivity on the association of FTO with overweight/obesity in our cohort; however, such a claim needs further investigation.

Although our study was not designed to assess the prevalence and distribution of obesity, our secondary findings suggest a similar prevalence of obesity and overweight rates to previous studies of the Kuwaiti population, with results showing females have higher rates of obesity $[1,32]$. Such findings may be attributed to genet- ic or lifestyle differences; however, further investigation on our population is required for adequate assessment.

\section{Conclusion}

We observed an association between the common FTO gene polymorphism (rs9939609) with overweight/ obesity. To the best of our knowledge this is the first reported association between FTO and BMI in the Kuwaiti population. Further replication studies in our population investigating the FTO rs9939609 are required to validate our findings. Other variants within the FTO gene and related genes regulated by the FTO should be investigated to further measure the impact of genetic factors on obesity. Studying the genetics of obesity in extremely obese populations can help us better understand the factors and mechanisms underlying the development of obesity in populations with similar obesogenic environments.

\section{Acknowledgement}

This research was partially supported and funded by the Kuwait University Research Administration, Project RM03/13, and the Kuwait Foundation for Advancement of Science, Project 201113021. The authors would like to thank Mrs. Reema Mathew and Mrs. Babitha G. Annice for their technical support in anthropometric measurements.

\section{Disclosure Statement}

There is no conflict of interest.

\section{References}

1 Ng M, et al: Global, regional, and national prevalence of overweight and obesity in children and adults during 1980-2013: a systematic analysis for the Global Burden of Disease Study 2013. Lancet 2014;384:766-781.

2 IDF: International Diabetes Federation Diabetes Atlas. Brussels, IDF, 2017.

3 Goodarzi MO: Genetics of obesity: what genetic association studies have taught us about the biology of obesity and its complications Lancet Diabetes Endocrinol 2017, Epub ahead of print.

4 Frayling TM, et al: A common variant in the FTO gene is associated with body mass index and predisposes to childhood and adult obesity. Science 2007;316:889-894.
5 Zhang M, et al: Age- and sex-dependent association between FTO rs9939609 and obesity-related traits in Chinese children and adolescents. PLoS One 2014;9:e97545.

6 Saldana-Alvarez Y, et al: Gender-dependent association of FTO polymorphisms with body mass index in Mexicans. PLoS One 2016; 11:e0145984.

7 Celis-Morales C, et al: Physical activity attenuates the effect of the FTO genotype on obesity traits in European adults: the Food $4 \mathrm{Me}$ study. Obesity (Silver Spring) 2016;24:962969.

8 Qi Q, et al: Dietary intake, FTO genetic variants, and adiposity: a combined analysis of over 16,000 children and adolescents. Diabetes 2015;64:2467-2476.
-9 Jia G, et al: Oxidative demethylation of 3-methylthymine and 3-methyluracil in singlestranded DNA and RNA by mouse and human FTO. FEBS Lett 2008;582:3313-3319.

10 Jia $G$, et al: $\mathrm{N}_{6}$-methyladenosine in nuclear RNA is a major substrate of the obesity-associated FTO. Nat Chem Biol 2011;7:885-887.

$\checkmark 11$ Gerken T, et al: The obesity-associated FTO gene encodes a 2-oxoglutarate-dependent nucleic acid demethylase. Science 2007;318: 1469-1472.

12 Loos RJ, Yeo GS: The bigger picture of FTO: the first GWAS-identified obesity gene. Nat Rev Endocrinol 2014;10:51-61.

13 Abdali D, et al: How effective are antioxidant supplements in obesity and diabetes? Med Princ Pract 2015;24:201-215. 
14 Physical status: the use and interpretation of anthropometry. Report of a WHO Expert Committee. World Health Organ Tech Rep Ser 1995;854:1-452.

15 Friedewald WT, et al: Estimation of the concentration of low-density lipoprotein cholesterol in plasma, without use of the preparative ultracentrifuge. Clin Chem 1972;18:499-502.

16 Qiagen: QIAamp DNA Mini Blood Mini Handbook. 2017.

17 Thermofisher: SNP genotyping analysis using TaqMan assays. Life Technologies, 2017.

$>18$ Rodriguez S, et al: Hardy-Weinberg equilibrium testing of biological ascertainment for Mendelian randomization studies. Am J Epidemiol 2009;169:505-514.

19 Gonzalez JR, et al: SNPassoc: an R package to perform whole genome association studies. Bioinformatics 2007;23:644-645.

20 Sentinelli F, et al: Association of FTO polymorphisms with early age of obesity in obese Italian subjects. Exp Diabetes Res 2012;2012: 872176.
21 Speliotes EK, et al: Association analyses of 249,796 individuals reveal 18 new loci associated with body mass index. Nat Genet 2010; 42:937-948.

22 Tan LJ, et al: Replication of 6 obesity genes in a meta-analysis of genome-wide association studies from diverse ancestries. PLoS One 2014;9:e96149.

23 Rodrigues GK, et al: A single FTO gene variant rs9939609 is associated with body weight evolution in a multiethnic extremely obese population that underwent bariatric surgery. Nutrition 2015;31:1344-1350.

24 Al-Sabah SK, et al: The efficacy of laparoscopic sleeve gastrectomy in treating adolescent obesity. Obes Surg 2015;25:50-54.

25 Kamura Y, et al: FTO Gene polymorphism is associated with type 2 diabetes through its effect on increasing the maximum BMI in Japanese men. PLoS One 2016;11:e0165523.
26 Bazzi MD, et al: Association between FTO, MC4R, SLC30A8, and KCNQ1 gene variants and type 2 diabetes in Saudi population. Genet Mol Res 2014;13:10194-10203.

27 Al-Sinani S, et al: Association of gene variants with susceptibility to type 2 diabetes among Omanis. World J Diabetes 2015;6:358-366.

28 Smemo S, et al: Obesity-associated variants within FTO form long-range functional connections with IRX3. Nature 2014;507:371375.

29 Srivastava A, et al: Association of FTO and IRX3 genetic variants to obesity risk in north India. Ann Hum Biol 2016;43:451-456.

30 Mittal B, et al: Is fat mass and obesity-associated (FTO) gene master regulator of obesity? Indian J Med Res 2016;143:264-266.

31 Alegria-Torres JA, et al: Epigenetics and lifestyle. Epigenomics 2011;3:267-277.

32 Awad AI, Alsaleh FM: 10-year risk estimation for type 2 diabetes mellitus and coronary heart disease in Kuwait: a cross-sectional population-based study. PLoS One 2015; 10:e0116742. 